\title{
Evaluation of the effect of the elastic abdominal strap, on the reduction of the respiratory restrictive syndrome in spinal cord, injured patients (C5 to T12) less than 6 months old
}

\begin{abstract}
Methods: 27 spinal cord injured subjects are included in the study to evaluate the effect of wearing of an elastic abdominal strap on the restrictive syndrome. Two measures are made: the measurements of staged thoracic amplification and the measurement of the respiratory functional exploration without and with abdominal strap.

Results: No effect on the respiratory functional exploration measurements ( $p$-value $>0.05$ ), significant increase in chest expansion at the axillary and xiphoid level $(p$-value $<0.05)$ and significant decreased in diaphragmatic amplification (p-value<0.05).

Discussion: The use of an abdominal strap does not improve the parameters of the respiratory functional exploration in spinal cord injured and opposes the mechanical and physiology of breathing by minimizing the action of the diaphragm and increasing the thoracic perimeters whatever it is the level of the spinal cord injury from C 5 to T12.

Conclusion: The use of an elastic abdominal strap in spinal cord injured patients does not improve respiratory functional exploration parameters and leads to an increase in axillary and xiphoidal thoracic amplification and to a decreased of the abdominal diaphragmatic one.
\end{abstract}

Keywords: Spinal cord injuries, elastic abdominal strap, spirometer, thoracic amplification, restrictive syndrome

\section{Introduction}

Paraplegia is a paralysis of both lower limbs resulting from a spinal cord injury, traumatic (accidental) most often, but sometimes may result from other medical causes. Every year in the world, between 250,000 and 500,000 people have spinal cord injuries (SCI) [1] (WHO, 2015).

In the lower cervical (C4 to $\mathrm{C} 7$ ) and upper dorsal (T1 to T6) medullar injury, the preservation of the diaphragmatic activity allows a ventilatory autonomy. Due to the disappearance of all or part of the intercostal muscles, and the total absence of the abdominal muscles, this autonomy is relative and precarious, there is a major paralysis of the main expiratory muscles that allow an effective cough (abdominal, intercostals). In lower dorsal medullar injury, (T6 to T12) ventilatory problems are minor and depend on the importance of the abdominal muscle paralysis $[2,3]$

Respiratory dysfunction resulting from paralysis remains a major cause of morbidity, mortality and economic charge [4]. It is the most common cause of death for these patients. This respiratory dysfunction is undervalued, if not totally ignored. These patients present a restrictive respiratory syndrome of varying importance depending on the level of the injury [3,5]. The deficit of the abdominal muscles, according to some studies is effectively compensated by the wearing of an elastic abdominal strap [6]. However, other studies have shown that the use of an abdominal strap does not improve respiratory function $[7,8]$. It seems interesting to know the effectiveness of the wearing of this elastic abdominal strap on the respiratory function of SCI patients.

We propose to study this impact on a population of 27 spinal cord injured patients,
Rola Tout ${ }^{1 *}$, Joseph

Maarrawi ${ }^{2}$ and Mohamad Halimi ${ }^{1,3}$

${ }^{1}$ Faculty of Medicine, Saint-Joseph University, Campus of Medical Sciences, Beirut, Lebanon. ${ }^{2}$ Laboratory of research in Neurosciences, PTS- Faculty of Medicine, Saint-Joseph University, Beirut, Lebanon.

Department of Neurosurgery, HotelDieu de France, Beirut, Lebanon ${ }^{3}$ Research training, Physical Therapy and Occupational Therapy, Berck-surMer, France.

*Author for correspondence:

rolatout@yahoo.com 
using the Respiratory Functional Exploration (RFE) and the staged measurement of thoracic extension, we would choose to compare the measures of the Vital Capacity (VC), Forced Expiratory Volume in one second (FEV1), Peak Expiratory Flow (PEF) and Tiffeneau's ratio, and measurements of inspiratory and expiratory thoracic amplification, at different levels, axillary, xiphoid and umbilical, with and without abdominal strap.

\section{Anatomo-physiology of the respiratory muscles}

The respiratory function provided by the respiratory system is used to supply the body with oxygen $\left(\mathrm{O}_{2}\right)$ to meet the needs of the organs that consume it for their activity. The expiration is passive whereas the inspiration requires a muscular effort to raise the thorax against the external resistances (gravity) and internal (muscular tone, the elasticity of the lungs, stiffness's, retractions and others $[9,10]$.

During inspiration, the inspiratory muscles, primarily the diaphragm and the external intercostals, increase the size and volume of the chest and the lungs allowing the passage of the ambient air to the alveoli [11]. The diaphragm, the main inspiratory muscle, by its contraction induces a cranial-caudal displacement of the phrenic center which increases the volume of the thoracic cage $[12,13]$.

While the abdominal muscles all have an expiratory activity, the diaphragm contracts by lowering the phrenic center, which increases the vertical diameter of the chest while exerting pressure on the abdominal viscera [14]. The abdominal muscles then intervene by forming a strap preventing the abdominal protrusion downwards and forwards and forming a solid point of support for the diaphragmatic contraction $[6,15]$.

\section{- Physiopathology}

Medullar lesions deprive of voluntary movement the muscles whose innervations are below the injury level. Thus, the higher the spinal cord injury, the greater is the extent of paralysis of the respiratory muscles. This is verifiable only for complete spinal cord lesions [11,14] (IMAGE 1).

\section{- Impact of paralysis of abdominal and intercostal muscles on respiratory function}

Severe diaphragmatic dysfunction will lead to a paradoxical breathing, gas exchange abnormalities, and sleep disturbances. The paralysis of the respiratory intercostal muscles will lead to respiratory insufficiency; the Vital Capacity (which can be reduced by $50 \%$ for quadriplegic subjects) varies according to the importance of intercostal muscle lesion and the flexibility of ribcage [16-18].

\section{- Respiratory functional exploration}

The respiratory functional explorations (RFE) gather all the examinations allowing measuring the quantifiable variables of the respiratory function. The normal expiratory flow-volume curve has the shape of a triangle, the inspiratory normal curve in the form of a semicircle (FIGURE 1). The values of the various respiratory parameters are greater than $80 \%$ of the theoretical values and the Tiffeneau's ratio is greater than $70 \%[12,19]$. The restrictive syndrome is characterized by a decrease in Total Lung Capacity (TLC) and an increase in Tiffeneau's ratio. Peak Expiratory Flows (PEF) is decreased in proportion to the decrease in TLC (FIGURE 2) [15,20].

\section{- Thoracic amplification perimeter}

The thoracic perimeter is used to judge the capacity of the thorax to distend [21]. The test is

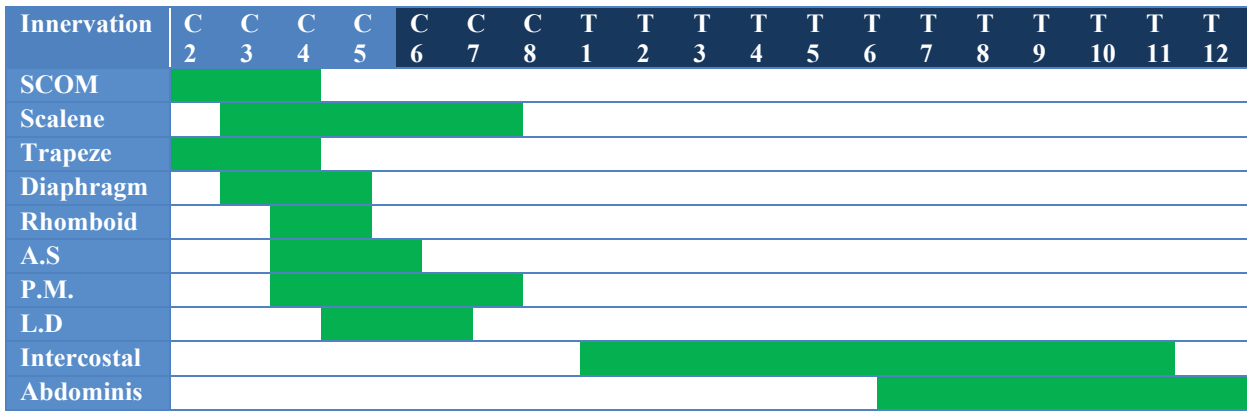

IMAGE 1: Metameric levels of respiratory muscles. SCOM: SternoCleido Mastoid, A.S: Anterior Serratus, P.M.: Pectoralis Major, L.D: Latissimus dorsal 
done by a tape measure. Measurement of upper and lower thoracic motility and diaphragmatic mobility are tested separately. An anatomical landmark corresponds to each of the three measurements levels:

- $4^{\text {th }}$ intercostal space to measure the upper ribs distention

- The xiphoid appendix to measure the lower ribs distention

- The umbilicus to measure the diaphragmatic distention [22]

The measurements are done in sitting position, inspiration and maximum expiration. The difference between the three measurements corresponds to the thoracic amplification [21]. The location of the umbilicus under abdominal strap will be done by tape measure referring to its distance from the jugular notch.

\section{Materials and Methods}

\section{- Population}

27 patients with a complete and symmetrical spinal cord injury were included in this study after verifications of the inclusion and exclusion criteria. TABLE 1 summarizes the clinical characteristics of patients. The average of the

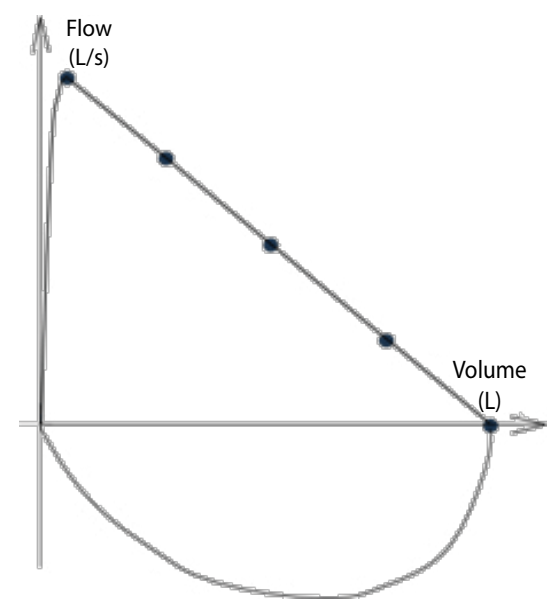

FIGURE 1: Normal flow-volume curve. age of the population is $23.1+3.89$ years $(18 \mathrm{yrs}$ $-30 \mathrm{yrs})$, with a sex ratio $\mathrm{M} / \mathrm{W}$ of $12.5 / 1$.

\section{Inclusion Criteria}

- Spinal Cord injuries (C5-T12) cooperative patient, between 18 and 35 years old, with a complete and symmetrical injury of the medulla, less than 6 months old, confirmed by a neurologist, presenting a restrictive syndrome on spirometric examination

\section{Non-inclusion Criteria}

- Associated respiratory diseases (asthma, bronchiectasis, Emphysema, rib fracture ...)

- Recent abdominal surgery (less than 6 months)

- Spasticity or significant retraction of the abdominal muscles (which limits thoracic expansion)

- Associated cerebral lesions (stroke, head trauma, epilepsy)

\section{- Experimental protocol}

Our experimental protocol is based on the use of the abdominal strap in the spinal cord

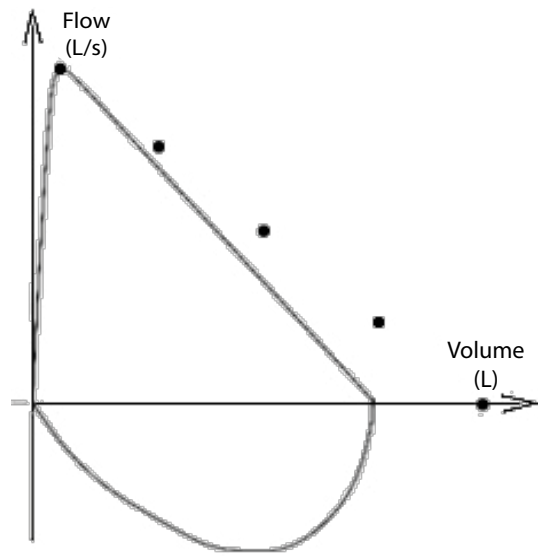

FIGURE 2: Flow-volume curve of the restrictive syndrome.

\begin{tabular}{|c|c|c|c|c|c|}
\hline & Levels of injury & Age & BMI & Sex & Smoking \\
\hline $\begin{array}{c}\text { Total } \\
27 \\
\text { patients }\end{array}$ & $\begin{array}{l}6 \text { patients }<\mathrm{T} 6 \\
21 \text { patients }>\mathrm{T} 6\end{array}$ & $23.11 \pm 3.89$ years & $\begin{array}{c}7.4 \% \text { underweight } \\
62.9 \% \text { normal weight } \\
14.8 \% \text { pre obesity } \\
3.7 \% \text { moderate obesity }\end{array}$ & $\begin{array}{l}7.5 \% \text { क } \\
92.5 \% \curvearrowright\end{array}$ & $\begin{array}{c}63 \% \text { smokers } \\
37 \% \text { non-smokers }\end{array}$ \\
\hline
\end{tabular}


injured patients in order to evaluate its effect on the different thoracic parameters.

FIGURE 3 presents the different phases of the experimental protocol.

The elastic strap we used is an abdominal lumbar band (Thuasne $e^{\bullet}$ Dynabelt Abdominal Belt) (FIGURES 4). In order to choose the right strap for each patient, an abdominal measurement was made of the abdominal perimeter in lying position at the level of the umbilicus with the tape measure. Quiet breathing is required of the patient; we measure the perimeter in an intermediate position between inspiration and expiration. It is essential that spirometric measurement is performed with the same tension of the strap. We arbitrarily adopted a tightening of about $10 \%$ elongation of the strap at the end of the expiration.

\section{Spirometric measurement tests}

An explanation of the interest of the examination was made to the patients before the execution of the tests, the initial and final balance sheets, carried out after oral agreement of the patients and signature of an informed consent, were made successively without and with the abdominal strap in a comfortable sitting position on a chair with backrest, with a minimum of 2 hours after the last meal. The final test, with the abdominal strap, was performed after 15 minutes of the initial test to allow a rest period to the patient. The results are compared with theoretical or predicted values, which are calculated based on the age, sex, and length of the patient's ethnic group [23]. The measurements are made with a nose clip and the patient's lips must surround the tip of the spirometer, in order to avoid any leaks.

\section{- Thoracic measurements}

In order to measure thoracic amplification, three successive measurements will be performed at two thoracic levels (axillary and xiphoidal) and an abdominal umbilicus level [21].

FIGURE 3:

Representation of the experimental protocol.
- 30 seconds of rest between each measurement.

- 1 minute of rest between each level.

- The best measurement of the three tests will be retained.

The anatomical level of the different measures :

- For the axillary perimeter: the tape measure is placed at the 4 th intercostal space (FIGURE 5).

- For the xiphoidal perimeter, the tape measure is placed at the level of the xiphoid appendix (FIGURE 6).

- For the umbilical perimeter, the tape measure is placed at the level of the umbilicus (FIGURE 7) [22].

The following instructions were given to patients:

- "Inhale slowly and push the meter tape while inflating the lungs" to measure maximum inspiration

- "Blow strongly by mouth while emptying the lungs to the maximum", for the measurement of maximum expiration [24]

\section{Statistical Analysis of the Data}

The results are expressed as the mean \pm standard deviation. We used the Xlstat 2009 statistical software. The average comparisons are

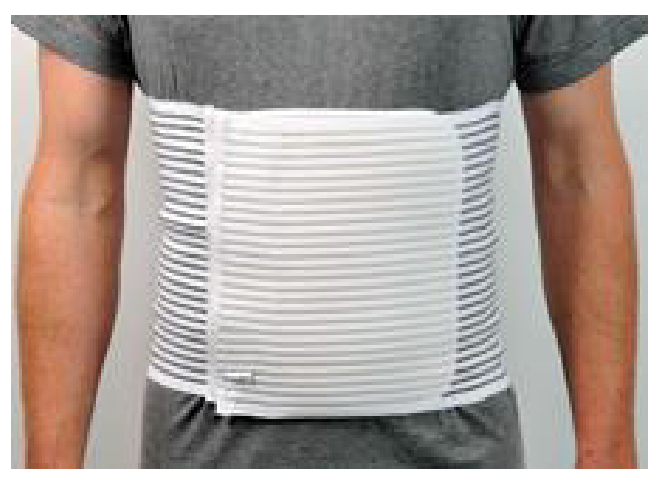

FIGURE 4: Abdominal Elastic Strap. 
made by T-test. A value of $\mathrm{p}<0.05$ is considered statistically significant.

\section{Results}

Following the spirometric and morpho dynamics assessments carried out with our population with spinal cord injured patients of varying levels (C5 to T12) whose injury is at least 6 months old, in the neuromuscular rehabilitation centers of North Lebanon, we obtained the following results:

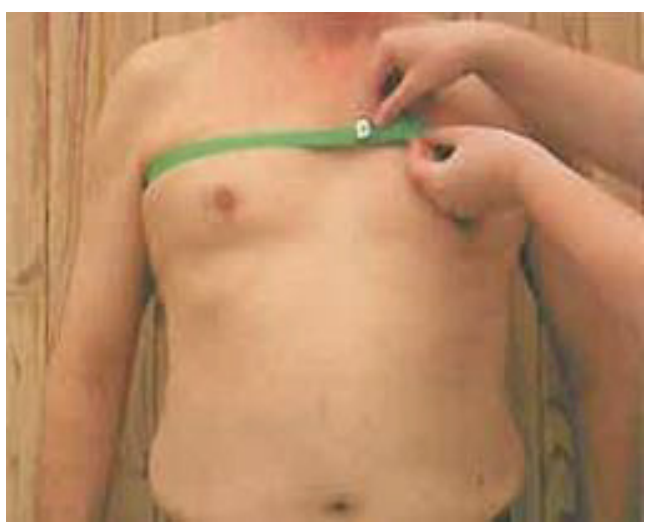

FIGURE 5: For the Axillary perimeter: the tape measure is placed at the 4th intercostal space

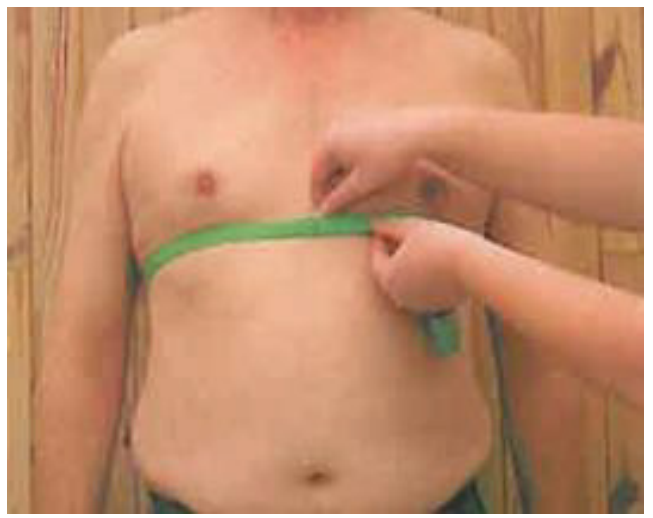

FIGURE 6: For the xiphoidal perimeter, the tape measure is placed at the level of the xiphoid appendix.

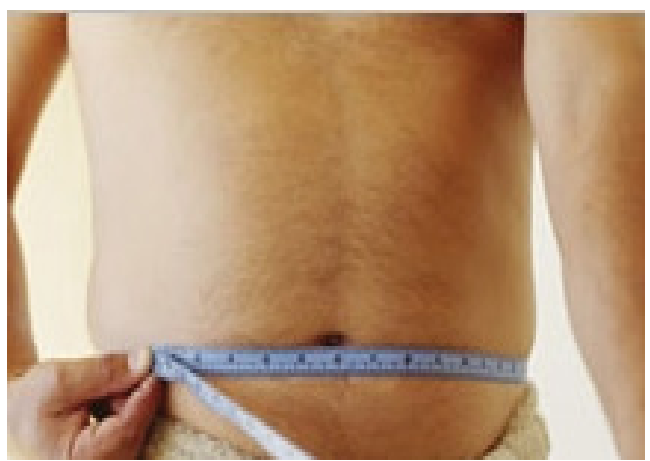

FIGURE 7: For the umbilical perimeter, the tape measure is placed at the level of the umbilicus.

\section{Results of the spirometric examination}

The examination has shown that the wearing of the abdominal strap in the spinal cord injured patient would not modify the spirometric measurements (FEV1, PEF, Tiffeneau's ratio, and FVC) and this regardless of the level of the injury.

There is no significant difference in FEV1, PEF, FVC and Tiffeneau's ratio, between spirometric measurements were done without the abdominal strap and those done with abdominal strap ((TABLE 2 and FIGURE 8).

\section{Evaluation of measurements of thoracic perimeters (axillary, xiphoidal and umbilical) without and with elastic abdominal strap}

A significant increase $(\mathrm{p}=0.0000010)$ between the axillary perimeter during the inspiratory $(102.09 \pm 14.66 \mathrm{~cm}$ without the strap and $103.48 \pm 14.50$ with strap and between the axillary perimeter during the exhalation time $(99.59 \pm 14.61 \mathrm{~cm}$ without the abdominal strap and $100.04 \pm 14.56 \mathrm{~cm}$ with abdominal strap $(\mathrm{p}=0.0027)$.

Significant increase $(\mathrm{p}=0.0000001)$ between the xiphoid thoracic perimeter during the inspiratory time $(100.93 \mathrm{~cm} \pm 13.31 \mathrm{~cm}$ without abdominal strap and $100.04 \mathrm{~cm} \pm 14.56$ $\mathrm{cm}$ with abdominal strap) and between the xiphoidal thoracic perimeter during expiratory time $(98.15 \mathrm{~cm} \pm 13.55 \mathrm{~cm}$ without abdominal strap and $98.67 \mathrm{~cm} \pm 13.82 \mathrm{~cm}$ with abdominal strap $(\mathrm{p}=0.0055)$.

A significant decrease $(\mathrm{p}=0.0000001)$ between the diaphragmatic abdominal perimeter during the inspiratory time $(105.5 \mathrm{~cm} \pm 16.57$ $\mathrm{cm}$ without the abdominal strap and $104.24 \mathrm{~cm}$ $\pm 17.03 \mathrm{~cm}$ with abdominal strap). A significant decrease $(\mathrm{p}=0.0058)$ between the diaphragmatic abdominal perimeter during the expiratory time $(103.11 \mathrm{~cm} \pm 16.96 \mathrm{~cm}$ without the abdominal strap and $102.44 \mathrm{~cm} \pm 17.20$ with abdominal strap) (FIGURE 9).

\section{Discussion}

\section{- Spirometric volumes}

All authors without exception agree that the use of abdominal strap induces real benefits in the spinal cord injured patient [25-27]; 
however, the majority of studies note a slight non-significant improvement in the parameters of the respiratory function test following the use of an abdominal strap [28,29].

The results of our study are consistent with studies of Wadsworth et al. [8], who demonstrated that the use of abdominal strap does not improve the parameters of respiratory function tests in spinal cord injured patients.

We divided our population into 2 groups: the first group of patients with an injury higher than T6, with absence of contraction of the abdominal muscle theoretically with the absence of an abdominal-diaphragmatic synergy and the second group of patients with an injury lower than T6 with partial presence of contraction of the abdominal muscles and presenting a partial presence of abdominal - diaphragmatic synergy.

The non-significance of the results obtained from the spirometric volumes with and without abdominal strap was identical for both groups of patients ( $<\mathrm{T} 6$ and $>\mathrm{T} 6$ ) and these results are consistent with the results of the meta-analysis by Wadsworth, which revealed evidence that the use of abdominal strap improves Vital Capacity (VC), but decreases Functional Residual Capacity (FRC).

We notice the reverse trends for the two groups of medullar injuries patients studied (paraplegic and quadriplegic). Indeed, with the elastic abdominal strap, quadriplegic patients improve their FVC by almost $5.6 \%$ and their Tiffeneau's ratio by $6.25 \%$. For paraplegic patients, the two parameters (FVC and

\begin{tabular}{|c|c|c|c|c|c|c|c|c|c|c|c|c|}
\hline & \multicolumn{3}{|c|}{ FEV 1} & \multicolumn{3}{|c|}{ PEF } & \multicolumn{3}{|c|}{ FEV } & \multicolumn{3}{|c|}{ Tiffeneau's Ratio } \\
\hline & $\begin{array}{c}\text { Entire } \\
\text { Population } \\
\end{array}$ & \begin{tabular}{|c|} 
injury $>$ \\
T6
\end{tabular} & $\begin{array}{l}\text { injury } \\
<\text { T6 }\end{array}$ & \begin{tabular}{|c|} 
Entire \\
Population
\end{tabular} & $\begin{array}{c}\text { injury> } \\
\text { T6 }\end{array}$ & $\begin{array}{l}\text { injury } \\
<\mathrm{T} 6\end{array}$ & \begin{tabular}{|c|} 
Entire \\
population
\end{tabular} & $\begin{array}{c}\text { Injury } \\
>\mathrm{T} 6\end{array}$ & injury $<\mathrm{T} 6$ & $\begin{array}{c}\text { Entire } \\
\text { population }\end{array}$ & $\begin{array}{c}\text { injury> } \\
\text { T6 }\end{array}$ & $\begin{array}{c}\text { injury< } \\
\text { T6 }\end{array}$ \\
\hline $\begin{array}{l}\text { Without } \\
\text { abdominal strap }\end{array}$ & $1.98 I \pm 0.69$ & $\begin{array}{c}1.27 I \pm \\
0.47\end{array}$ & $\begin{array}{c}2.381 \pm \\
0.48\end{array}$ & \begin{tabular}{|c|}
$4.16 \mathrm{l} / \mathrm{s} \pm$ \\
1.32 \\
\end{tabular} & $\begin{array}{c}2.46 \mathrm{l} / \mathrm{s} \\
\pm 0.39\end{array}$ & $\begin{array}{c}4.86 \mathrm{l} / \mathrm{s} \pm \\
0.83\end{array}$ & $2.54 I \pm 0.65$ & $\begin{array}{c}2.11 I \pm \\
0.61\end{array}$ & $\begin{array}{c}2.831 \pm \\
0.51\end{array}$ & $\begin{array}{c}0.78 \% \pm \\
0.17\end{array}$ & $\begin{array}{c}0.64 \% \pm \\
0.25\end{array}$ & $\begin{array}{c}0.83 \% \pm \\
0.08\end{array}$ \\
\hline $\begin{array}{l}\text { With abdominal } \\
\text { strap }\end{array}$ & $2.01 \mathrm{I} \pm 0.68$ & $\begin{array}{c}1.26 \mathrm{I} \pm \\
0.56\end{array}$ & $\begin{array}{l}2.35 I \pm \\
0.45\end{array}$ & $\begin{array}{c}4.19 \mathrm{l} / \mathrm{s} \pm \\
1.39\end{array}$ & $\begin{array}{l}2.73 \mathrm{l} / \mathrm{s} \\
\pm 1.39\end{array}$ & $\begin{array}{c}4.88 \mathrm{l} / \mathrm{s} \pm \\
0.84\end{array}$ & $2.46 I \pm 0.73$ & $\begin{array}{c}1.99 I \pm \\
0.74\end{array}$ & $\begin{array}{c}2.79 I \pm \\
0.57\end{array}$ & $\begin{array}{c}0.79 \% \pm \\
0.17\end{array}$ & $\begin{array}{c}0.68 \% \pm \\
0.25\end{array}$ & $\begin{array}{c}0.83 \% \pm \\
0.1\end{array}$ \\
\hline
\end{tabular}

\section{Evaluation of different parameters of the spirometric measurements}

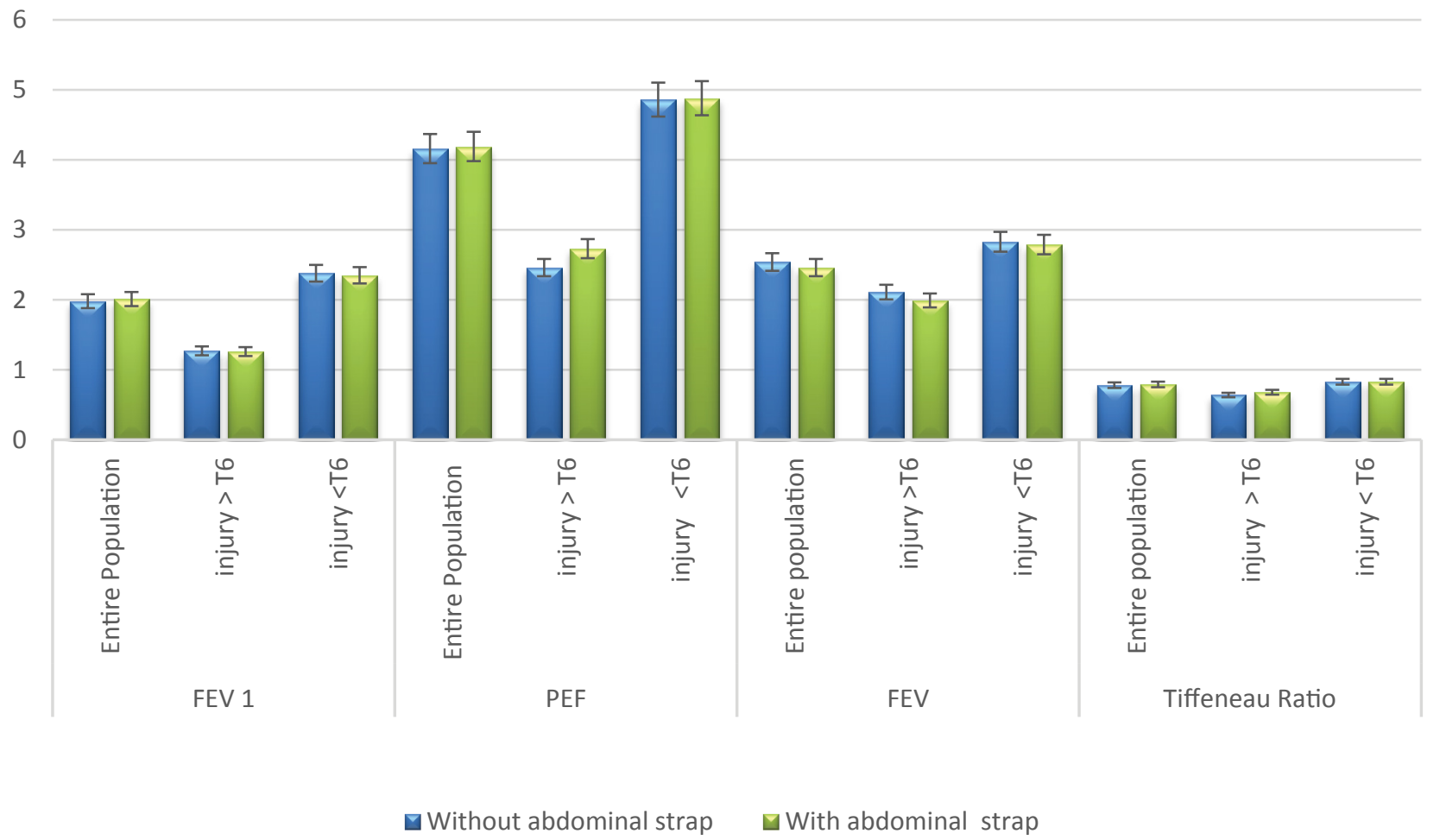

FIGURE 8: Evaluation of FEV1, PEF, FVC and Tiffeneau's ratio measurements with and without abdominal strap. 


\section{Evaluation of thoracic amplification perimeters}

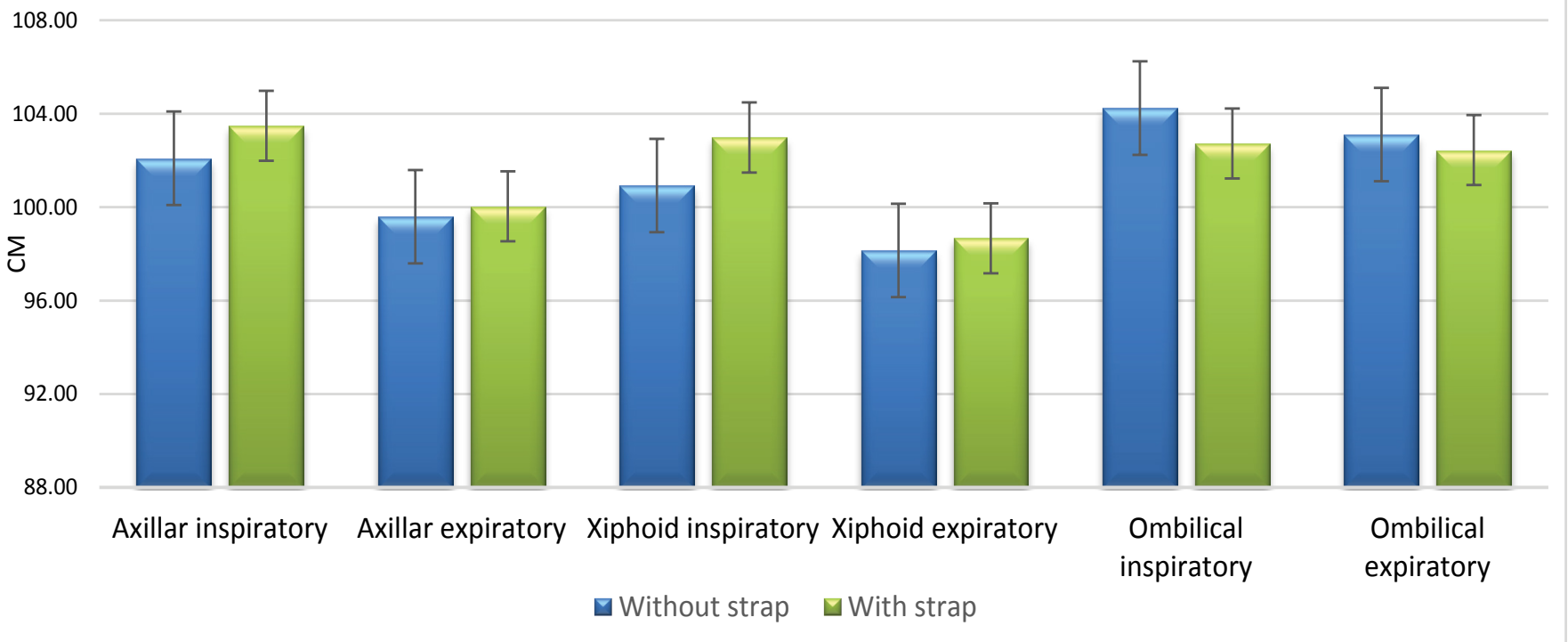

FIGURE 9: Evaluation of thoracic amplification perimeters with and without abdominal strap.

Tiffeneau's ratio) vary negligibly for the two tests (1.4\% for FVC and $0 \%$ for Tiffeneau's ratio); this could possibly be explained by the presence in these patients of a paradoxical breathing and a costal draw in spinal cord injured patients higher than T6. Both groups of patients showed only a negligible increase in FEV1 (by almost $1.6 \%$ compared to the value without strap).

Similarly, in the group of quadriplegic subjects (injury> T6), we note that patients (A.J) and (M.H), have a decrease in FEV1 with strap, unlike all others. These patients, who are heavy smokers, have a severe restrictive syndrome accompanied by an obstructive syndrome during initial measurements, which explains why the wearing of an abdominal strap limits the expiratory flow of these patients.

The practical difficulty encountered during this work is the tightness of the strap for the (AC) patient, who had a very small abdominal perimeter, 65 centimeters, the waist strap size " 1 " is the smallest and is normally put in place for a patient. The abdominal perimeter is ranging from 66 to $80 \mathrm{~cm}$. However, it remained too big for this patient. We have exceptionally tightened the strap for about $15 \%$ from the original length. The result for this quadriplegic subject (injury at T6), was a decrease in all parameters of the Respiratory Functional Exploration (RFE). We can suppose that by placing a strap on this quadriplegic subject, we decrease the abdominal thoracic balance, which results in a decrease of the respiratory parameters with strap. It should be noted that all paraplegic patients report being embarrassed by using the strap.

\section{Measurement of thoracic extension}

Axillary and xiphoidal volume differences increased significantly, which should be beneficial for the patient's breathing, but the problem was that this increase in thoracic amplification was accompanied by a decrease in the difference in inspiratory and expiratory diaphragmatic expansion. This antagonizes the respiratory physiology and exaggerates the non-synergy between the thorax and abdominal respiratory function in these patients thus increasing their apical and thoracic breathing. These results are consistent with the results of the West et al. 2014 [30], concerning the usefulness of wearing abdominal strap on the improvement of the respiratory function during exercise in sports quadriplegics (without making a thoracic amplification measurements) that the abdominal strip displaces the breathing but reduces the volumes lungs without influencing the flow limitation.

We believe that the increase in axillary and xiphoid thoracic perimeters concomitant with the decrease in diaphragmatic amplification 
is related to thoracic solicitation, beyond respiratory physiology in the spinal cord injured patient. These patients, who already have a restrictive syndrome and an inability to achieve significant physiological thoracic amplification, would be forced to perform, during the wearing of the elastic abdominal strap, a purely thoracic and non-physiologic apical breathing which could go in the direction that may increase their restrictive syndrome.

These results support our initial hypothesis that abdominal strap would not improve the restrictive syndrome of spinal cord injured patient.

We must, therefore, accept that the problem present in these subjects is not a question of the capacity of the abdominal muscles to contain the abdominal viscera which could be compensated by an elastic abdominal strap.

\section{Conclusion}

We can conclude from this experimental study that the use of an abdominal strap in spinal cord injured patients (C5-T12) less than 6 months old does not lead to a significant improvement in respiratory functional exploration measurements regardless of the level of the medullar injury. In addition, it shifts patients' breathing to the higher thoracic levels and significantly minimizes the difference in diaphragmatic amplification. The abdominal strap provides no respiratory benefit in subjects with paralysis of the respiratory muscles (especially the abdominal muscles), and its use leads to worsening of the purely thoracic paradoxical breathing.

Following this study, it is thought that other therapeutic solutions should be considered to improve, if possible, the respiratory parameters of the spinal cord injured patients. 


\section{References}

Van den Berg MEL, Castellot JM, Mahillo-Fernandez I. Incidence of spinal cord injury worldwide. Neuroepidemiology. 34, 184-192 (2010).

De Troyer A. Actions of the respiratory muscles. The Thorax. 535-63 (1995).

Hayot M. Les muscles respiratoires sont-ils des muscles squelettiquescomme les autres? Revue. Des. Maladies. Respiratoires. 34, 525-590 (2017).

David C, David F, Lesley M. Medical complications during acute rehabilitation following spinal cord injury, current experience of the model systems. Arch. Phys. Med. Rehabil. 80 (11), 1950-1956 (2012).

AndradaL, De Vito EL. Functional respiratory evaluation in patients with high traumatic spinal injury. Medicina. 61(1), 529-534 (2001).

DeVivoMJ. Long-term medical complications after traumatic spinal cord injury: a regional model systems analysis. Arch. Phys. Med. Rehabil. 80(11), 14021410 (1999).

Bodin P. Effects of abdominal binding on breathing patterns during breathing exercises in persons withtetraplegia. Thorax. 41, 940-945 (1986).

Wadsworth BM, HainesTP, Cornwell PL. Abdominal binder use in people with spinal cord injuries: a systematic review and meta-analysis. Spinal Cord. 47(4), 274-285 (2009).

Fauroux, B.T, Pourquoi consacrer un Séminaire d'approfondissement et de Perfectionnement en Pneumologie aux muscles respiratoires ? Revue des Maladies Respiratoires. 22 (1), 5-7 (2005).

Anna L, HudsonSimon C, Gandevia Jane E. Control of human inspiratory motoneurones during voluntary and involuntary contractions. Respir. Physiol.
Neurobiol. 179(1), 23-33 (2011).

Baydur A, Adkins R, Milic-Emili J. Lung mechanics in individuals with spinal cord injury: effects of injury level and posture. Appl. Physiol. 90(2), 405-411 (2001).

Marchand E, Les muscles respiratoires Anatomie, physiologie, exploration fonctionnelle et importance en physiopathologie respiratoire. La Lettre du Pneumologue. II (6), (1999).

StrausC, Comment estcontrôlée la fonction des muscles respiratoires? Revue des Maladies Respiratoires. 22, 19-28 (2005).

Forner J V. Lung volumes and mechanics of breathing in tetraplegics. Paraplegia. 18 (2), 258-266 (1980).

Similowski. T. Édition française des recommandationsconjointes de l'American Thoracic Society et de l'EuropeanRespiratory Society pour l'Exploration de la Fonction des Muscles Respiratoires. Revue des Maladies Respiratoires. 2(1), 447-449 (2004).

ChenCF, Lien I. Respiratory function in patients with spinal cord injuries: effects of posture. Paraplegia. 28(2), 81-86 (1990).

Fugl-Meyer AR. Effects of respiratory muscle paralysis in tetraplegic and paraplegic patients. Scand. J. Rehabil. Med. 3(4), 141-150 (1971).

BrasJ. Noninvasive determination of inspiratory pressure in patients with spinal cord injury: What is the best method? Pneumol. 35(3), 256-60 (2009).

AnkeA. Lung volumes in tetraplegic patients according to cervical spinal cord injury level. Scand. J. Rehabil. Med. 157(1), 33-42 (1998).

Quanjer P. H, Tammeling GJ, Cotes JE, Pedersen OF. Volumes pulmonaires et débits ventilatoires forcés. Revue des maladies respiratoires. 11(3), 5-40 (1994).
Maurel E. Des méthodes de mensuration delacage thoracique. Bulletins et Mémoires de la Société d'Anthropologie de Paris. 10, 345-368 (1887).

Gouilly P, Reggiori B, Gnos P.L, Schuh O, Muller K. A propos de la mesure de l'ampliation thoracique. Kinésithérapie La Revue. 9(88), 49-55 (2009).

Fujiwara T, Hara Y. Expiratory function in complete tetraplegics. Am. J. Phys. Med. Rehabil. 78(5), 464-469 (1999).

Préfaut C, Racineux JL, Sergysels R. L'exploration fonctionnelle respiratoire en pratique pneumologique. Rev. Mal. Respir. 3, 323-466 (1986).

Christopher R, Campbell G, Robert E, Lee M. Effects of abdominal binding on cardiorespiratory function in cervical spinal cord injury. Respir. Physiol. Neurobiol. 180(2-3), 275-282 (2012).

EstenneM, Muylem A. Effects of abdominal van strapping on forced expiration in tetraplegic patients. $A m$ J Respir. Crit. Care Med. 25(2), 73-77 (1993).

Boaventura CM. Effect of an abdominal binder on the efficacy of respiratory muscles in seated and supine tetraplegic patients. Physiotherapy. 89, 290-295 (2003).

Brooke M, Terry P, Petrea L, Leanne $\mathrm{T}$, Jennifer $\mathrm{D}$, Abdominal binder improves lung volumes and voice in people with tetraplegic spinal cord. Arch Phys Med Rehabil. 93(12), 2189-2197 (2012).

Christopher R, Campbell G, Victoria L. Effects of abdominal binding on fieldbased exercise responses in Paralympic athletes with cervical spinal cord injury. J. Sci. Med. Sport. 17(4), 351-355 (2014).

West C. Effect of abdominal binding on respiratory mechanics during exercise in athletes with cervical spinal cord injury. J. Appl. Physiol. 117(1), 36-45 (2014). 